\title{
A clinical study of early manifestations of dengue fever and outcome in children
}

\section{Dr. Neelagiri Udaya Kumar}

\author{
Professor, Department of Pediatrics, Prathima Institute of Medical Sciences, Naganoor, Karimnagar \\ Address for communication: Dr. Neelagiri Udaya Kumar, Professor of Pediatrics, Flat No -710, Prithvi Block, My home Navadwepa, \\ Madhapur-500081, Hyderabad.Email: drudayn@yahoo.co.in \\ Mobile: 9849255544 \\ DOI : 10.47799/pimr.0802.06
}

\section{ABSTRACT}

Background: In the past few decades the incidence, distribution, and clinical severity of Dengue have increased dramatically. Population growth in the tropics provides many susceptible hosts. Uncontrolled urbanization leads to inadequate management of water and waste, providing a range of large water stores and disposable, non-biodegradable containers that become habitats for the larvae. Children are now being increasingly affected by this disease. We in this present study tried to study clinical, hematological, and hepatic dysfunction in the early course of the disease.

Methods: The present prospective observational study was conducted at Prathima Institute of Medical Sciences, Nagnur, Karimnagar. $\mathrm{N}=75$ children between the age group of 6 months to 15 yrs presenting with symptoms and signs suggestive of dengue fever as per WHO criteria were included in the study. Complete history and clinical examination were done. Investigations were performed as per the proforma.

Results: From $n=75$ children $n=56$ (75\%) belongs to dengue with warning signs and severe dengue whereas $n=19$ cases ( $25 \%$ ) belong to mild dengue group. the symptoms recorded out of $n=75(100 \%)$ children presented with fever as the predominant complaint followed by vomiting (49\%), Retroorbital pain (28\%), Abdominal pain (26\%), Edema (25\%), Bleeding (19\%) and rashes (12\%), body ache $(8 \%)$. The range of platelet count at admission was $4,000-98,000 / \mathrm{cmm}$, with a mean value of 53,000 ( $P>0.05)$. The WHO criteria of a low platelet count of $<100,000 / \mathrm{cmm}$ were seen in all cases $(100 \%)$.

Conclusion: in children, importance should be given to symptoms like fever, vomiting, bleeding, musculoskeletal pain, flushing, and abdominal pain. If these are associated with hepatomegaly, positive tourniquet test, low platelet count, and elevated liver enzymes, a strong possibility of dengue to be considered, especially during an epidemic. During the epidemic, dengue should be considered on the differential diagnosis of any child presenting with fever.

Keywords: children, Dengue fever, Early clinical manifestations Introduction

Dengue fever is an acute febrile viral disease characterized by sudden onset of fever in 3-5 days, intense headache, myalgia, retro-orbital pain, anorexia, gastrointestinal disturbances, and rash. Dengue is caused by viruses that are classified as a flavivirus, it includes four serotypes DENV 1, 2, 3 , and 4 . The viruses are transmitted to humans by the bite of infective mosquitoes, mainly Aedes aegypti. The incubation period is 4-7 days but ranges from 3 to 14 days. The disease is now endemic in most tropical, subtropical countries. Severe dengue is characterized by increased vascular permeability, hypovolemia, and abnormal blood clotting mechanisms. ${ }^{[1]}$ Dengue ranks as the most important mosquito-borne viral disease in the world. Current estimates report that at least 112 countries are endemic for Dengue and about $40 \%$ of the world populations (2.5-3 billion people) are at risk in tropics and sub-tropics. ${ }^{[2-4]}$ Annually 100 million cases of dengue fever and half a million cases of dengue hemorrhagic fever occur worldwide. $90 \%$ of DHF subjects are less than 15 years of age. Early recognition and prompt initiation of treatment are vital if disease-related morbidity and mortality are to be limited. ${ }^{[5]}$ This study aims to fill in some of these gaps by describing the complications and clinical outcomes of children presenting with dengue infection, particularly with the application of WHO guidelines for classification and management of dengue fever. Despite the seriousness of the disease, many clinicians are still not fully aware of WHO guidelines in the classification and management of dengue fever. The severity of the illness is determined by various risk factors such as age, pre-existing illness, infecting serotype, secondary dengue infection. ${ }^{[3]} \mathrm{A}$ second infection with a different serotype leads to a more severe form of the disease than the primary infection. ${ }^{[6]}$ Studies have shown that the infecting serotype determines the severity of the illness with DEN 2 and DEN 3 causing the most severe form of the disease. ${ }^{[7]}$ The timely appropriate management of dengue fever can considerably reduce both morbidity and mortality. Despite advances in diagnostic modalities and treatment strategies, there is limited published Indian data on dengue fever in the pediatric population. Hence we in the current study tried to evaluate the clinical, hematological, and hepatic dysfunction in the early course of dengue in pediatric cases presenting to our tertiary care teaching institute. 


\section{Material and Methods}

The present prospective observational study was conducted at Prathima Institute of Medical Sciences, Nagnur, Karimnagar. $\mathrm{N}=75$ children between the age group of 6 months to 15 yrs presenting with symptoms and signs suggestive of dengue fever as per WHO criteria were included in the study. Institutional Ethical committee permission was obtained for the study after duly following the protocol. Written consent was taken from parents/parents/guardians before enrolling in the study. Complete history and clinical examination were done. Investigations were performed as per the proforma. The results were tabulated and correlated. The outcome was recorded in every subject. The criteria defined by WHO as mentioned previously were followed the inclusion of subjects into the study.

\section{Inclusion criteria:}

- All children of age group 6 months to $15 y e a r s$

- clinical signs and symptoms suggestive of dengue fever as per WHO criteria

- IgM or NS1 positive cases

\section{Exclusion criteria:}

- Patients negative for NS1Ag or IgM antibody.

- Age $<6$ months and $>15$ years

- Those diagnosed with viral fevers other than Dengue

\section{Investigations:}

The following investigations were performed in all the children which included Blood $\mathrm{Hb} \%, \mathrm{PCV}, \mathrm{TLC}, \mathrm{DLC}$, Platelet count, Peripheral blood smear, hemoparasites PT, APTT Liver function test SGOT, SGPT, Serum Alkaline Phosphatase, Serum albumin, Chest X-ray Abdominal ultrasound. Dengue serology for NS1 and IgM all the seventy-five children registered in the study were treated at PICU, as per the WHO algorithm on dengue fever. They were discharged after complete recovery as per guidelines. All the available data was recorded in the MS Excel spreadsheet and analyzed using SPSS version 19 on the widows' format.

\section{Results}

The mean age was 5 yrs 4 months, the youngest in the series was 6 months and the eldest was 15 yrs old. Out of the $\mathrm{n}=75$ children, $\mathrm{n}=39$ were male and $\mathrm{n}=36$ were female. The ratio $M: F=1.1: 1$. Out of $n=75$ cases, $n=43(57.3 \%)$ were registered in the months of September and October which are considered peak times of dengue. From $n=75$ children $n=56$ (75\%) belongs to dengue with warning signs and severe dengue whereas $n=19$ cases (25\%) belong to mild dengue group. In the urban area $n=54$ cases $(72 \%)$ and in the rural area $n=21$ cases $(28 \%)$ were documented in this study. Based on Kuppuswamy scale out of $n=75$ children, $n=52$ children $(69 \%)$ belong to the middle class.

Table 1: Demographic profile of the cases included in the study

\begin{tabular}{|c|c|c|c|c|c|}
\hline $\begin{array}{c}\text { Age group } \\
\text { (Years) }\end{array}$ & No. of cases & Percentage & Mild dengue & Severe dengue & $\begin{array}{c}\text { Dengue with } \\
\text { warning signs }\end{array}$ \\
\hline$<1$ & 5 & 6.67 & 0 & 2 & 3 \\
\hline $1-4$ & 34 & 45.33 & 10 & 12 & 12 \\
\hline $4.1-8$ & 21 & 28 & 4 & 10 & 7 \\
\hline $8.1-12$ & 12 & 16 & 4 & 5 & 3 \\
\hline$>12$ & 2 & 2.67 & 1 & 1 & 1 \\
\hline Total & 75 & 100 & 19 & 30 & 26 \\
\hline
\end{tabular}

In the symptoms recorded out of $n=75$ (100\%) children presented with fever as the predominant complaint followed by vomiting (49\%), Retro-orbital pain (28\%), Abdominal pain

(26\%), Edema (25\%), Bleeding (19\%) and rashes (12\%), body ache $(8 \%)$ shown in table 2.

Table 2: Analysis of symptomatology presented by the cases in the study

\begin{tabular}{|l|c|c|c|c|c|c|}
\hline \multicolumn{1}{|c|}{ Symptom } & No. of cases & Percentage & Mild dengue & $\begin{array}{c}\text { Severe } \\
\text { dengue }\end{array}$ & $\begin{array}{c}\text { Dengue with } \\
\text { warning signs }\end{array}$ & p-value \\
\hline Fever & 75 & 100 & 19 & 30 & 26 & 1.998 \\
\hline Vomiting & 37 & 49.33 & 10 & 14 & 13 & 0.559 \\
\hline Retro-orbital pain & 21 & 28 & 7 & 10 & 4 & 1.225 \\
\hline
\end{tabular}




\begin{tabular}{|l|c|c|c|c|c|c|}
\hline \multicolumn{1}{|c|}{ Symptom } & No. of cases & Percentage & Mild dengue & $\begin{array}{c}\text { Severe } \\
\text { dengue }\end{array}$ & $\begin{array}{c}\text { Dengue with } \\
\text { warning signs }\end{array}$ & p-value \\
\hline Abdominal pain & 20 & 26.67 & 4 & 11 & 5 & 0.959 \\
\hline Edema & 19 & 25.33 & 7 & 10 & 2 & $<0.05^{*}$ \\
\hline Bleeding manifestations & 14 & 18.67 & 0 & 12 & 2 & $<0.05^{*}$ \\
\hline Rash & 9 & 12 & 6 & 2 & 1 & $<0.05^{*}$ \\
\hline Headache & 7 & 9.33 & 3 & 1 & 3 & 1.22 \\
\hline Altered sensorium & 8 & 10.67 & 1 & 4 & 3 & 0.987 \\
\hline seizers & 7 & 9.33 & 1 & 3 & 3 & 0.366 \\
\hline Body ache & 6 & 8 & 8 & 3 & 3 & $<0.05$ \\
\hline Diarrhea & 4 & 53.33 & 2 & 2 & 0 & 0.551 \\
\hline
\end{tabular}

* Significant

The mean duration of fever was 6.6 days and ranges from 2-15 days the skin bleeds were the common manifestation in $n=14$ cases $(37.8 \%)$ followed by GIT bleeding like melena $\mathrm{n}=12$ cases $(32.4 \%)$ and hematemesis 5 cases $(13.5 \%)$. the

\begin{tabular}{|l|c|c|}
\hline \multicolumn{1}{|c|}{ Duration of fever (days) } & No. of cases & Percentage \\
\hline $1-3$ & 14 & 18.7 \\
\hline $4-5$ & 23 & 30.7 \\
\hline $6-10$ & 32 & 42.6 \\
\hline$>10$ & 6 & 8.0 \\
\hline Total & 75 & 100 \\
\hline
\end{tabular}

On CNS examination agitations and restlessness was observed in many cases $n=8(10.6 \%)$ children had altered sensorium and another 8 children had convulsions. Although convulsions are known to occur in dengue fever the exact cause has not been established one child presented with coma The

\begin{tabular}{|c|c|c|c|c|c|}
\hline $\begin{array}{l}\text { Types of } \\
\text { bleeding }\end{array}$ & No. of cases & Percentage & Mild dengue & Severe dengue & $\begin{array}{l}\text { Dengue with } \\
\text { warning signs }\end{array}$ \\
\hline Skin & 14 & 37.8 & 0 & 12 & 2 \\
\hline Epistaxis & 2 & 5.4 & 1 & 0 & 1 \\
\hline Hematemesis & 5 & 13.5 & 0 & 3 & 2 \\
\hline Melena & 12 & 32.4 & 1 & 8 & 3 \\
\hline Puncture site & 1 & 2.7 & 0 & 0 & 1 \\
\hline Multiple & 3 & 8.1 & 0 & 1 & 2 \\
\hline Total & 37 & 100 & 2 & 24 & 11 \\
\hline
\end{tabular}

bleeding manifestations were more in dengue with warning signs, severe dengue group than mild dengue group given in table 3

Table 3: Showing the duration of fever in cases at the time of

hematocrit ranged from $20-49 \%$ with a mean value of $34.7 \%$. No child presented with less than $20 \%$ of hematocrit. The suggested cut off hematocrit value for dengue with warning signs ( $>36.3 \%$ ) as per Indian standards was seen in 33 children (44\%). The range of total leukocyte count varied from 2,500- 
The range of platelet count at admission was 4,000 $98,000 / \mathrm{cmm}$, with a mean value of 53,000 ( $P>0.05)$. The WHO criteria of a low platelet count of $<100,000 / \mathrm{cmm}$ was seen in all cases $(100 \%)$ given in (table 5 ) Bleeding was more frequent with low platelet count in dengue with warning signs, severe dengue group compared to mild dengue group however it was statistically not significant $(P<0.05)$.

Table 5: The mean platelet counts of cases at the time of admission

\begin{tabular}{|c|c|c|c|c|}
\hline $\begin{array}{c}\text { Platelet count in } \\
\text { thousand/mm3 }\end{array}$ & No. of cases & Mild dengue & Severe dengue & $\begin{array}{c}\text { Dengue with } \\
\text { warning signs }\end{array}$ \\
\hline$<20,000$ & 11 & 2 & 5 & 4 \\
\hline $20,001-30,000$ & 5 & 0 & 2 & 3 \\
\hline $30,001-50,000$ & 25 & 3 & 5 & 12 \\
\hline $50,001-75,000$ & 14 & 6 & 6 & 3 \\
\hline $75,000-1,00,000$ & 20 & 8 & 30 & 6 \\
\hline Total & 75 & 19 & & 26 \\
\hline
\end{tabular}

The range for SGOT was 21-4510 IU/It with a mean of 252.1 IU/It. SGPT - Normal 5-45 IU/It. The range for SGPT was 18-1667 IU/It with a mean of $109.8 \mathrm{IU} / \mathrm{It}$. The significant fourfold rise of both transaminases seen $n=23$ (30.7\%) children. The range for ALKP was 8-532 IU/It with a mean of $121.9 \mathrm{lU} / \mathrm{lt}$. The range for serum albumin was $1.2-3.8 \mathrm{gm} \%$ with a mean of $2.80 \mathrm{gm} \%$.

Serum albumin of $<2.5 \mathrm{gm} \%$ was seen in $32 \%$ of children, more in DWS and SD group. Chest $x$-ray was done in all the $n=75$ cases, in $n=39$ children $(52 \%)$ there was pleural effusion. In the DWS and SD groups, the number of cases was more compared to the mild dengue group. $(X 2=11.7, P<0.05)$ It was statistically significant. Ultrasonography was done in $\mathrm{n}=13$ cases, $\mathrm{n}=5$ cases showed right-sided pleural effusion, bilateral pleural effusion in $n=2$ cases, and ascites in $n=6$ cases.

\section{Discussion}

Dengue fever is the most important arboviral infection and has become a major global public health problem. In India, epidemics are becoming more frequent. The involvement of younger age groups and an increase in the frequency of epidemics are indicators of higher incidence of infection. Classical dengue fever is an acute febrile illness but in a small percentage of Dengue infection, a more severe form of the disease occurs. Early recognition and meticulous management are very important to save precious lives from this killer disease. Although a high incidence of degree has been described in children very few studies have been exclusively on them. The 5-11 year age group dominated the present study, accounting for $42 \%$ of the total dengue cases reported to our hospital. The youngest child in the present study was 6 months old, 1 boy and 1 girl. Gomber et al; ${ }^{8]}$ conducted the study at New Delhi in the year 2001 with a result of $78.9 \%$ were children. Narayanan et al; ${ }^{[9]}$ in south India found $45 \%$ of dengue cases were found in children. The maximum numbers of cases were found in the months of September and October similar observations were made by Anuradha et al; ${ }^{[10]}$ where the maximum number of cases was from September to November and Gomber et al; ${ }^{[8]}$ found maximum cases from July to October. Most of the Indian studies have registered the monsoon and post-monsoonmonths as the period of maximum incidence, the breeding season of Aedes Aegyptus. Dengue has always been linked to rapid urbanization, explaining its high incidence among middle-class families. The present study had $52(69 \%)$ of the children from middle-class families, as previously observed in other studies by WHO meta-analysis ${ }^{[11]}$, Rasul et al; ${ }^{[12]}$ in the present study fever (100\%) was the predominant (100\%) symptoms followed by vomiting (47\%), retro-orbital pain (28\%), abdominal pain (26\%), bleeding (19\%), Rashes (12\%). Similar observations were made by other studies. $\left[{ }^{[10,11]}\right.$ In the present study bleeding manifestations Hemetemesis was found in $21 \%$ of cases Kumar et al; ${ }^{[13]}$ Lucknow 2000 found $31.2 \%$ cases with Hematemesis. Rahman et al; ${ }^{[14]}$ in Bangladesh found $46 \%$ cases with Melena. Narayanan et al; ${ }^{[9]}$ found $66 \%$ of cases with Melena. Features of shock were noted in 8 cases (10.6\%) at admission, of whom 4 (5.3\%) had severe dengue and $4(5.3 \%)$ of them succumbed. Nimmannitya et al; ${ }^{[15]}$ SEAR $196935 \%$, Srinivasa et al; ${ }^{[16]}$ in New Delhi found severe dengue in $19 \%$. The mean platelet in the present study was $53,000 /$ $\mathrm{cmm}$ with the range of 4000 to $98000 /$ cumm. The platelet counts at the admission were neither an indicator of prognosis nor of bleeding tendencies or progression of the disease. These suggest that other factors like platelet dysfunction or disseminated intravascular coagulation may have a role in bleeding in dengue fever cases. However, studies that include only DHF cases show the correlation between low platelet count and bleeding manifestations70. The studies by Gomber et al; ${ }^{[8]}$ and Narayanan et al; ${ }^{[9]}$ have documented the same opinion. ${ }^{[17,18]}$ But platelet count provides a very useful means of diagnosis at the screening level. Hence the platelet count 
was a sensitive indicator for diagnosis but it did not correlate with the outcome. Bleeding manifestations are more frequent with low platelet count. Incidentally, 2 children had co-existing plasmodium falciform ring forms, in the peripheral smear. They were treated for malaria simultaneously. Out of the 75 children, 69 (92\%) recovered without any sequelae. 6(8\%) children who presented in late stages succumbed.

\section{Conclusion}

Within the limitations of the present study, it can be concluded that in children, importance should be given to symptoms like fever, vomiting, bleeding, musculoskeletal pain, flushing, and abdominal pain. If these are associated with hepatomegaly, positive tourniquet test, low platelet count, and elevated liver enzymes, a strong possibility of dengue to be considered, especially during an epidemic. During the epidemic, dengue should be considered on the differential diagnosis of any child presenting with fever. Bleeding tendencies should be closely watched. The treatment of dengue is mainly supportive, but the early institution and meticulous monitoring are the cornerstones for a positive outcome.

\section{REFERENCES}

1. Gibbons RV, Vaughn DW. Dengue: an escalating problem BMJ, 2002; 324:1563-66

2. Bhatt S, Gething PW, Brady OJ, Messina JP, Farlow AW, Moyes $\mathrm{CL}$, et al. The global distribution and burden of dengue. Nature. 2013;496(7446):504.

3. World health organization, editor. Comprehensive guidelines for prevention and control of dengue and dengue hemorrhagic fever. Rev and expanded. ed. New Delhi, India: World Health Organization Regional Office for South-East Asia. 2011:196. Available at:

h t t p:// w w w. searo.who.int/entity/ vector_borne_tropical_diseases/documents/ SEAROTPS60/en/. [accessed on 15 Jan 2020]

4. Gupta N, Srivastava S, Jain A, Chaturvedi UC. Dengue in India. Indian J Med Res.2012;136(3):373.

5. Malavige GN, Fernando S, Fernando DJ, Seneviratne SL. Dengue viral infections. Postgrad Med J 2004; 80:588-601.

6. Guzman MG, Halstead SB, Artsob H, Buchy P, Farrar J, Gubler DJ, Hunsperger E, Kroeger A, Margolis HS, Martinez E: Dengue: a continuing global threat. Nat Rev Microbiol 2010, 8(12 Suppl):S7-16.

7. Balmaseda A, Hammond SN, Perez L, Tellez Y, Saborio SI, Mercado JC, Cuadra R, Rocha J, Perez MA, Silva S: Serotype-specific differences in clinical manifestations of dengue. Am J Trop Med Hyg 2006, 74(3):449-456.

8. Gomber S, Ramachandran VG, Kumar S, Agarwal, Gupta P, Gupta P et al Hematological observations as diagnostic markers in dengue hemorrhagic fever - a reappraisal. Indian Pediatr 2001; 38: 477-481.

9. Narayanan M, Arvind MA, Thilothammal N, Prema R, Sargunam Rex CS, et al. Dengue Fever Epidemic in Chennai - A Study of Clinical Profile and Outcome. Indian Pediatr 2002; 39: 1027-1033.

10. Anuradha S, Singh NP, Rizvi SN, Agarwal SK, Gur R, Mathur MD. 1996 the outbreak of dengue hemorrhagic fever in Delhi, India. Southeast Asian J Trop Med Public Health. 1998; 29: 503-6.

11. World Health Organization. Prevention and Control of Dengue and Dengue Hemorrhagic Fever. WHO SEARO Publication No.29, New Delhi 1999.

12. Rasul $\mathrm{CH}$, Ahasan HAMN, Rasid AKMM, Khan MRH. Epidemiological Factors of Dengue Hemorrhagic Fever in Bangladesh. Indian Pediatr 2002; 39: 369-372.

13. Kumar ND, Tomar V, Singh B, Kela K. Platelet transfusion practice during Dengue fever epidemic. Indian J Pathol Microbiol. 2000; 43: 55-60.

14. Rahman M, Rahman K, Siddique AK, Shoma S, Kamal AH, Ali KS et al. First outbreak of Dengue hemorrhagic fever, Bangladesh. Emerg Infect Dis. 2002; 8:738-40.

15. Nimmannitya S. Dengue and Dengue Hemorrhagic fever in the South-East Asian Regions. Am J Trop Med Hyg. 1969; 18: 954-971.

16. Srivastava VK, Suri S, Bhasin A, Srivastava L, Bharadwaj $M$. An epidemic of Dengue hemorrhagic fever and Dengue shock syndrome in Delhi: a clinical study. Ann Trop Pediatr 1990; 10: 329-334.

17. Kabra SK, Jain Y, Pandey RM, Madhulika, Singhal T, Tripath Pet al. Dengue hemorrhagic fever in children in the 1996 Delhi epidemic. Trans R Soc Trop Med Hyg. 1999; 93: 29458.

18. Mohan B, Patwari AK, Anand VK. Hepatic dysfunction in childhood Dengue infection. J Trop Pediatr. 2000; 46: 4043.

How to cite this article : Neelagiri Udaya Kumar. A clinical study of early manifestations of dengue fever and outcome in children. Perspectives in Medical Research 2020; 8 (2):1519. DOI : 10.47799/pimr.0802.06

Sources of Support: Nil, Conflict of interest: None declared 